\title{
L'utilisation pédagogique des tablettes numériques au CM2 à Niamey (Niger) : réussite scolaire des élèves et avantages pédagogiques
}

\author{
Mahamadou Halilou Garba, Université Abdou Moumouni \\ Nana Aicha Goza, Université Abdou Moumouni
}

\section{DOI : 10.51186/journals/ed.2020.10-2.e351}

\section{Résumé}

Le but de cette étude est de faire l'état des lieux de l'utilisation des tablettes numériques et de leur impact sur la réussite scolaire des élèves de CM2 à Niamey, au Niger. Pour ce faire, nous avons constitué un échantillon composé de deux groupes d'écoles : l'un numérique (avec 153 élèves et trois enseignantes) et l'autre non numérique (avec 115 élèves et trois enseignantes). Une méthodologie de type quasi-expérimentale, dans le sens de comparer deux groupes en termes de résultats dans un contexte de salle de classe, a été déployée dans cette étude. Au niveau des élèves, ce sont les notes qui sont comparées. Au niveau des enseignantes, c'est le sentiment d'auto-efficacité. De plus, un questionnaire sur l'utilisation du numérique en classe a été administré aux élèves du groupe expérimental. Les résultats montrent que les élèves des écoles numériques, avec 07,64/10 de moyenne, réussissent mieux que les élèves des écoles non numériques avec 06,79/10. L'analyse de la variance montre également qu'il y a une relation significative entre la nature des établissements et les moyennes des élèves. L'utilisation des tablettes numériques impacte donc positivement la réussite scolaire des élèves. Le sentiment d'auto-efficacité des enseignantes n'a lui pas d'impact.

Mots-clés : TIC, résultats d'apprentissage, pédagogie, enseignement primaire, Niger

\section{Abstract}

The purpose of this study is to examine the use of digital tablets and their impact on the academic success of CM2 (last year of primary school) pupils in Niamey, Niger. The study's sample comprised two groups of schools: one that uses digital tablets (153 pupils and 3 teachers) and one that does not (115 pupils and 3 teachers). A quasi-experimental methodology was used to compare the two groups in terms of classroom achievement. With regard to pupils, we compared the quarterly grade averages of the two groups of schools. With regard to teachers, we measured their perceptions of self-efficacy. Results show that pupils in schools that use digital tablets performed better than pupils in schools that did not, with yearly grade averages of $7.64 / 10$ and $6.79 / 10$, respectively. The analysis of variance also shows that there is a significant relationship between the nature of the schools and the pupils' grade 
averages. The use of digital tablets thus has a positive impact on pupils' classroom achievement, whereas self-efficacy of teachers does not.

Keywords: ICT, learning outcomes, pedagogy, primary education, Niger

\section{INTRODUCTION}

En Afrique francophone, les technologies éducatives ont fait leur entrée à l'école depuis les années 1960. II s'agissait d'une expérimentation de la radio et de la télévision scolaires dans les pays de l'Afrique de l'Ouest notamment la Côte d'ivoire, le Togo, le Sénégal et le Niger. L'objectif de cette expérimentation a été de rehausser le taux de scolarisation (Gado, 2011). À la fin de celle-ci, le bilan a été mitigé dans la plupart des pays concernés (Awokou, 2007). Cependant, dans le cas du Niger, Egly (1986), Maes (1969) et Saivre (1998), cités par Gado (2011) affirment que cette expérience a été plutôt positive. II explique que les raisons de son abandon ne sont pas liées à une quelconque inefficacité mais qu'elles sont à rechercher dans les divergences ayant caractérisé les rapports entre les acteurs en présence.

À la suite de la radio et de la télévision scolaires, le Niger a initié, avec l'appui de ses partenaires au développement, divers projets sur l'intégration de technologies modernes en éducation. C'est le cas de l'« Université Virtuelle Africaine » du «Campus Numérique Francophone », des projets Écoles numériques de brousse pour l'introduction du tableau blanc interactif et du Projet d'alphabétisation des adultes par le biais des téléphones portables (Coulibaly, 2009 ; Gado, 2011).

Nonobstant ces initiatives, l'usage des Technologies de l'information et de la communication (TIC) n'est pas officiellement inscrit dans les programmes d'enseignement des cycles primaire et secondaire. Celles-ci ne sont donc ni enseignées, ni intégrées comme outils pédagogiques au niveau de ces cycles (Coulibaly, 2009 ; Kouawo, 2011). Cependant, les enseignants et les élèves font régulièrement usage de ces ressources numériques dans le cadre des activités scolaires souvent en dehors de l'école (Aboubacar \& Adamou, 2018). C'est dans ce contexte que le projet «Education numérique » d'Orange/Niger a vu le jour à partir de 2014. Fruit du partenariat entre la société de télécommunication Orange et le Ministère de l'enseignement primaire, de l'alphabétisation, de la promotion des langues nationales et de l'éducation civique (MEP/A/PLN/EC), ce projet a doté plusieurs écoles primaires (75 au total) en tablettes numériques pour permettre leur usage dans le processus enseignement/apprentissage. Les tablettes dudit projet intègrent divers contenus (écrits, sons, vidéos) dont les manuels scolaires du programme officiel de l'enseignement primaire. II faut souligner que la même société a conduit, un an auparavant, une expérimentation similaire dans deux collèges (urbain et périurbain) de Niamey. Cette dernière a fait l'objet de recherche-bilan réalisée par Baron, et al. (2015). Ces auteurs ont relevé un certain nombre de plus-values liées à l'usage des tablettes, en particulier l'accroissement du niveau de motivation chez les apprenant-es ayant bénéficié 
des équipements numériques à travers le projet. Aussi, notre objectif est de vérifier la corrélation entre l'utilisation de ces tablettes numériques et la réussite scolaire des élèves du cycle primaire (CM2).

\section{CONTEXTE ÉDUCATIF ET PROBLÈME DE RECHERCHE}

Les contextes éducatifs africains traversent de profondes crises de nos jours. Celles-ci s'expriment sous forme de chute des performances scolaires dont l'intensité varie d'un pays à l'autre. A titre d'exemple, l'évaluation du Programme d'analyse des systèmes éducatifs de la CONFENEM (PASEC) (2014) cité par Tiemtoré (2016, p. 299) révèle qu'à l'échelle des pays membres de la Conférence des ministres de l'Éducation des pays ayant le français en partage (CONFEMEN), « 59\% des enfants de 5ème année du primaire ont un niveau de mathématique faible ». Cette moyenne cache des disparités car dans ce taux, le Benin compte 60,2\%, le Burkina 41\%, la Cote d'Ivoire 73,1\%, le Sénégal 41,2\% et le Niger 92,4\%.

Pour pallier aux difficultés que rencontre son système éducatif notamment celles relatives aux taux de scolarisation, d'achèvement et de faibles performances des apprenants-es, l'État du Niger a entrepris des reformes depuis plusieurs années. Des projets et des programmes ont ainsi été développés : le Programme décennal de développement de l'éducation (PDDE, 20032013), le Programme sectoriel de l'éducation et de la formation (PSEF, 2014-2024) et le Programme transitoire sectoriel de l'éducation et de la formation (PTSEF, 2020-2023).

Ces réformes correspondent aux engagements internationaux auxquels le Niger a souscrit tels que l'atteinte des Objectifs du millénaire pour le développement (OMD) et les Objectifs de développement durable (ODD). Elles s'inscrivent également dans une logique nationale du Programme de Développement économique et social (PDES, 2010) qui englobe l'ensemble des secteurs d'intervention de l'État. L'élan mondial de la scolarisation universelle donné à la Conférence de Jomtien 1990 a permis l'élaboration du PDDE dont l'un des objectifs était de rehausser le taux brut de scolarisation du Niger qui était de 42 \% en 2002 (PASEC, 2014). Le pari est réussi pour ce programme au niveau du taux d'accès au système éducatif formel qui est passé de 42 \% en 2002 à plus de 76,2 \% en 2016 (MEP/A/PLN/EC, 2016).

Cependant, force est de constater que ce progrès n'a été obtenu qu'au plan quantitatif. Sur le plan qualitatif (développement des compétences et acquisition des connaissances), les résultats sont plutôt faibles au niveau du cycle primaire. Les problèmes d'acquisition des connaissances s'installent et s'aggravent comme le mentionne le MEP/A/PL/EC (2018a) :

Le rapport de l'évaluation nationale 2011 des acquis scolaires a montré que le nombre d'élèves en situation d'échec scolaire a accru considérablement au cours préparatoire (CP), au cours élémentaire deuxième année (CE2) et au cours moyen deuxième année (CM2) en français comme en mathématiques. Dans le premier cas (français), les élèves en difficultés d'apprentissage sont estimés à $51,40 \%$ au CP, 67,13\% au CE2 et 72,67\% au CM2. En 
mathématiques, ces proportions sont respectivement estimées à 33,70\%, 68,80\% et 72,39\%. (p. 3)

Les résultats de l'enquête PASEC (2014) vont dans le même sens et font état de plus de neuf élèves sur dix du Cours préparatoire deuxième année (CP2) ayant des difficultés d'apprentissage en langue. Et près de trois-quarts des élèves de ce même niveau ont des difficultés d'apprentissage en mathématiques (RESEN Niger, 2018). La même source indique qu'en fin de scolarité primaire (CM2), plus de 90\% des élèves ont des performances en lecture en deçà du seuil suffisant de compétence. Une proportion similaire rencontre des difficultés en mathématiques.

Pour prendre en charge les insuffisances de la période du PDDE, notamment par rapport à la qualité des acquis scolaires, le PSEF (2014) a été élaboré et mis en œuvre. Ce dernier se voulant holistique, prend en compte l'ensemble du système éducatif (éducation formelle et non formelle). L'un des principaux objectifs du PSEF est ainsi de relever la qualité des acquis scolaires dans l'ensemble du système éducatif, particulièrement au niveau du cycle primaire. Pour atteindre ce but, le PSEF se propose d'introduire les TIC et l'enseignement à travers les langues nationales en guise de solutions permettant de rehausser la qualité de la formation des enseignant-es et d'améliorer les compétences des apprenant-es.

En effet, au Niger, la langue officielle est le français, c'est-à-dire que tous les enseignements du primaire à l'université sont dispensés dans cette langue étrangère. L'usage de cette dernière comme langue d'enseignement pourrait expliquer les faibles performances des élèves nigériennes. Une étude récente menée au plan national a ainsi mis en évidence la faiblesse des performances des écoles traditionnelles (exclusivement francophones) par rapport aux écoles bilingues (Halilou Garba, et al., 2020). L'idée d'enseigner en langues nationales a vu le jour depuis 1972 avec la création des écoles bilingues expérimentales. Plus tard avec la mise en œuvre du PDDE (2004) et la refondation du curriculum, l'enseignement en langue nationale est institué au primaire. Toutefois, il faut souligner le manque de volonté politique dans la mise en œuvre effective de cette décision. Cependant avec l'introduction de l'approche par situation (APS), le Niger a introduit les enseignements en langues nationales aux niveaux $\mathrm{Cl}$ et $\mathrm{CP}$ à travers 500 écoles pilotes (PASEC, 2014).

Quant à l'importance de l'intégration des TIC à l'école, objet du présent travail, Karsenti, et al. (2010), cités par Coulibaly (2020), les perçoivent comme un moyen pouvant favoriser et faciliter les changements dans les structures du système d'éducation, les programmes d'études, le perfectionnement du personnel enseignant et l'apprentissage des élèves. En contexte nigérien, Aboubacar \& Adamou (2018) soutiennent également que l'intégration des TIC en éducation pourrait être une solution aux multiples défis de l'école nigérienne. Cet optimisme est soutenu par des travaux ayant mis en évidence le potentiel cognitif des outils numériques (Depover, et al., 2007) ainsi que les qualités ergonomiques et pédagogiques de la tablette (Karsenti, 2015). Ce qui fait dire à Supper, et al. (2019) que les tablettes sont des outils dotés d'un fort potentiel 
pédagogique. En effet, l'apprentissage avec la tablette permet à l'enseignant-es d'utiliser au mieux la pédagogie différenciée et le travail collaboratif. Les élèves d'une même classe peuvent suivre des parcours d'apprentissage différents et développer des compétences en fonction du rythme d'acquisition de chaque élève. C'est dire que l'usage pédagogique du numérique peut être un moyen efficace pour améliorer les résultats scolaires des élèves.

Par contre, certains auteurs comme Moeglin (2017) soutiennent que le manuel numérique ne contribue pas à produire les effets escomptés chez les acteurs de l'enseignement apprentissage. Pour lui, on peut reprocher aux supports didactiques numérisés moult limites telles que: le manque d'attractivité, la préférence des enseignants pour des documents non didactiques, pour les manuels traditionnels, pour les ressources des sites collaboratifs et le défaut de données scientifiques fiables concernant la plus-value des innovations dans le domaine. Dans la même logique, Inaudi (2017) pense que l'avènement du numérique et de l'informatique à l'école n'est que la conséquence du basculement des systèmes éducatifs dans l'économie du marché.

C'est dire que les controverses sur l'influence du numérique dans l'apprentissage persistent encore aujourd'hui. Cela rappelle le célèbre débat qui a opposé Clark \& Kozma (Dessus, 2015). Clark, sceptique vis-à-vis de l'effet des médias en milieu scolaire, soutient que ces derniers n'ont aucune influence sur l'apprentissage alors que Kozma, fervent technophile, affirme que les médias influencent positivement les activités cognitives des apprenants.

II faut préciser que dans le contexte du Niger, un constat se dégage sur la question des TIC : ces dernières sont plus que jamais présentes dans le quotidien des nigérien-nes et, de plus en plus, elles sont présentes à l'école (primaire, secondaire et supérieure). On peut penser que cette présence accrue est la preuve d'une certaine utilité aussi relative soit-elle. La question actuelle consiste donc à se demander comment utiliser le numérique en faveur des apprentissages ? Et, de ce point de vue, plusieurs aspects entrent certainement en ligne de compte selon le type d'acteur (enseignant et élève) considéré dans le système d'éducation.

Pour ce qui est des enseignant-es, l'intégration du numérique en milieu scolaire requiert de leur part une certaine qualification dans le domaine techno-pédagogique. Cette dernière leur permet de mesurer les exigences techniques et organisationnelles des TIC lors de la conception/usage des supports numériques tout en tenant compte des objectifs pédagogiques (Karsenti, 2012). L'usage du numérique en éducation n'ayant pas que des avantages, il implique aussi de nombreux défis à relever (Karsenti, 2015 ; Karsenti \& Fievez, 2013).

Comment relever ces défis dans un contexte où les enseignant-es ne manquent pas seulement des compétences techno-pédagogiques, mais du minimum requis pour exercer leur métier? C'est-à-dire les compétences professionnelles. Les résultats de l'évaluation des enseignants contractuels effectuée par le MEP/A/PLN/EC (2018b) illustrent bien le manque de compétences de ces derniers. En effet, sur les 6979 enseignants contractuels ayant subi le 
test : seuls 33,0\% d'entre eux ont obtenu la moyenne ou plus (10,0 à 20/20) ; 45,8\% ont obtenu une note comprise entre 5 et 10/20 ; 13,6\% des notes comprises entre 3 et 5/20 et enfin $7,5 \%$ ont obtenu des notes situées entre 0 et 3/20. En outre, il ressort de ces résultats que les enseignant-es contractuel-les non formé-es réussissent autant que ceux qui ont reçu une formation initiale dans les écoles normales.

Ces résultats expliquent en partie les faibles performances des élèves déplorées par le ministère en charge de l'enseignement primaire. Par conséquent, on peut constater aisément l'effet maître dans l'acquisition des connaissances et le développement des compétences des apprenant-es. C'est pour pallier ce problème de faibles rendements internes de son système éducatif que l'État nigérien et ses partenaires ont introduit les TIC à l'école en dépit des nombreux obstacles y afférant (Coulibaly, 2012).

En dehors du manque de compétences d'ordre strictement professionnel relevé chez les enseignants, il existe d'autres obstacles à l'intégration effective des TIC dans l'éducation au Niger. II s'agit notamment :

- Du déficit de formation adéquate à l'usage pédagogique des TIC à l'intention des enseignants du primaire ;

- De l'accessibilité des TIC avant, pendant et après les heures de classes est aléatoire ;

- Du déficit d'investissement de ressources financières pour la formation, le soutien technique, l'achat et la maintenance des équipements ;

- Du déficit de soutien administratif ;

- Du déficit technologique lié d'une part à la faible couverture en énergie électrique et en connexion internet et, d'autre part à l'absence d'un personnel qualifié pour assurer le suivi technique et la production des ressources didactiques numériques adaptées.

La présence des équipements technologiques en salle de classe ne garantit donc pas de façon systématique les avantages pédagogiques liés au numérique (Karsenti, et al., 2019). L'intégration du numérique en éducation requiert plusieurs compétences qui vont de la compétence pédagogique à la compétence relative aux contenus à enseigner en passant par la maîtrise technique de l'outil numérique ainsi que la disponibilité et la fiabilité de l'énergie électrique ou de la connexion internet. C'est pourquoi Karsenti, et al. (2019) expliquent que :

Pour qu'un enseignant intègre durablement et efficacement les technologies en classe, il est impératif pour lui de saisir la complexité des enjeux, bien au-delà d'une simple maitrise de certains outils. Or la plupart des initiatives d'intégration pédagogique se contentent d'arrimer équipements technologiques, formation des enseignants et contenus d'intégration en classe. (p. 8)

En contexte nigérien des études ont essayé de comprendre comment se présente cette complexité de l'intégration des TIC à l'école. Cependant, il est rare que ces recherches abordent le cas des tablettes numériques en tant qu'outils pédagogiques. C'est pourquoi la présente étude s'intéresse à l'utilisation des tablettes numériques dans le cadre du projet 
Éducation numérique. Elle s'intéresse plus précisément à l'impact de l'utilisation des tablettes numériques sur la réussite scolaire des élèves du CM2. Partant du contexte de l'étude en lien avec la faiblesse des acquis scolaires des élèves et l'utilisation des TIC comme solution envisagée, nous formulons la question de recherche suivante : quel est l'impact de l'utilisation de la tablette numérique sur la réussite scolaire des élèves ?

\section{CADRE THÉORIQUE}

Ce travail s'inscrit dans l'approche de la genèse instrumentale de Rabardel (1995) qui établit une différence entre l'objet (artéfact) et l'instrument. Par genèse instrumentale, il faut comprendre le processus par lequel se développe ou s'élabore l'instrument. Tout objet n'est pas un instrument. II le devient lorsque se développe autour de lui un ensemble de « conduite d'utilisation organisée " appelé schèmes. Ses schèmes d'utilisation sont soit le produit d'une construction sociale, soit le résultat d'une construction individuelle du sujet. La genèse instrumentale est un mouvement à double sens. Du sujet vers l'artefact et de l'artefact vers le sujet. Rabardel (1995) appelle le premier mouvement l'instrumentalisation et le second l'instrumentation.

L'instrumentalisation implique les connaissances antérieures du sujet et les représentations qu'il mobilise pour satisfaire un besoin. II accommode, adapte l'outil ou l'objet à ses besoins en enrichissant parfois ses propriétés au-delà des prévisions de sa/son concepteur/trice.

Quant à l'instrumentation, c'est le processus inverse grâce auquel les propriétés de l'artéfact agissent sur le sujet qui modifie ses schèmes, donc une partie de ses connaissances et représentations. L'action de l'artéfact sur le sujet permet la construction des nouveaux schèmes, des nouveaux savoirs.

Pour ce qui est de l'utilisation des tablettes numériques par les élèves, on peut dire à la lumière de la théorie de Rabardel qu'il s'engage un mouvement réciproque entre les élèves et les tablettes numériques. C'est de ce double mouvement « sujet-objet » et « objet-sujet » que l'on appelle genèse instrumentale que va naitre l'instrument «tablette ». De ce rapport entre les sujets (élèves) et les objets (tablettes) émergent et se construisent des savoirs.

Cette partie présente les recherches sur l'utilisation pédagogique des tablettes numériques dans le contexte occidental où l'on peut parler d'une certaine intégration de celles-ci à l'école. S'ensuit l'historique de l'insertion des TIC en contexte scolaire nigérien et la part des tablettes dans celui-ci.

\subsection{Les tablettes à l'international}

Depuis l'avènement des tablettes numériques à l'école, des études se sont penchées sur leur impact éducatif. Comment cet outil peut-il contribuer au développement des apprentissages ? 
Pour ce faire il faut comprendre les facteurs organisationnels, pédagogiques et techniques qui expliquent les usages et les non-usages des tablettes tactiles (Villemonteix \& Khaneboubi, 2012, 2013). II faut aussi connaitre le processus d'appropriation collective des artéfacts éducatifs pendant l'interaction entre les éléments en présence, à savoir les élèves (apprenants), l'adulte (enseignant), l'artefact (tablettes) et le savoir (Bernard, et al., 2012). Les avantages et les défis de l'utilisation pédagogique des tablettes ont été soulignés par Karsenti \& Fievez (2013). Ces derniers ont énuméré 15 avantages et 7 défis que nous citons ici par ordre d'importance. Les 15 avantages sont les suivants : 1 . Motivation accrue des élèves ; 2 . accès à l'information ; 3. Portabilité de l'outil ; 4. annotation de documents PDF facilitée ; 5. organisation du travail plus aisée ; 6. Qualité des présentations réalisées par les élèves ; 7. Qualité des présentations réalisées par l'enseignant ; 8. Collaboration accrue entre les élèves, puis aussi entre les élèves et l'enseignant ; 9. Créativité soutenue ; 10. Variété des ressources présentées (images, vidéos, applications, etc.) ; 11. Possibilité d'aller à leur rythme pour les élèves ; 12. Développement de compétences informatiques chez les élèves ; 13. Développement de compétences informatiques chez les enseignants ; 14. Expérience de lecture bonifiée ; 15. Économie de papier pour les enseignants (p. 34).

Les 7 défis soulignés par Karsenti \& Fievez (2013) sont : 1. Distraction des élèves ; 2. Planification de cours ; 3. Gestion de travaux des élèves ; 4. Méconnaissance des ressources disponibles par les enseignants ; 5. Sous-usage des livres électroniques ; 6. L'apprentissage de l'écriture ; 7. Nuisance à la réussite scolaire par l'effet de distraction (p. 34).

Dans la même perspective, Colegnesi \& Balleux (2018) notent trois plus-values dans les pratiques pédagogiques à l'aide des tablettes numériques : la différenciation pédagogique, la créativité et la construction de synthèse. Ces plus-values ne déterminent pas forcement une amélioration des rendements scolaires des élèves, comme l'indiquent Supper, et al. (2019) qui ont mesuré les rendements scolaires en lien avec la fréquence d'utilisation des tablettes à travers la motivation autodéterminée. II ressort de cette étude qu'une utilisation plus fréquente de la tablette numérique pour effectuer des tâches éducatives prédit un meilleur rendement scolaire en mathématiques et en français (mais pas en anglais). En dépit de cette situation on relève une grande satisfaction des élèves concernant l'utilisation pédagogique des tablettes (Lestrohan, et al., 2016).

A contrario, des auteur-es comme Dauphin (2016) montrent que les tablettes numériques produisent plutôt un impact négatif sur les résultats scolaires des élèves. Son étude, l'une des rares à évaluer les impacts quantitatifs de la réussite scolaire, montre que l'utilisation de l'iPad en classe s'est traduite par une baisse générale des résultats scolaires.

Ce bref parcours de la littérature sur l'utilisation pédagogique des tablettes numériques permet d'affirmer à la suite de Karsenti et Fievez (2013) que ce ne sont pas les technologies qui déterminent les avantages pédagogiques mais plutôt les usages qu'on en fait. 


\subsection{Les recherches sur les TIC au Niger}

Au Niger, les recherches sur l'utilisation du numérique en éducation ont débuté il y a une vingtaine d'années. Les premières recherches se sont intéressées à l'enseignement supérieur. En effet les technologies numériques ont intégré l'école à travers l'enseignement secondaire et supérieur. Notons qu'auparavant la télévision scolaire avait été expérimentée à l'école primaire. C'est ainsi que Galy \& Dané (2003) ont traité des obstacles et des solutions à l'appropriation des Nouvelles technologies de l'information et de la communication (NTIC, dénomination consacrée à l'époque) à l'université de Niamey. Ils ont souligné les efforts déployés par I'université pour faciliter l'accès des enseignants-es et des étudiants-es aux NTIC. Cependant, plus de dix ans après, l'utilisation des ressources numériques rencontre encore des obstacles comme le déclare Moussa Sagayar (2016) dans une étude sur l'utilisation des ressources numériques au sein de quatre universités publiques du Niger. Cette étude montre la nécessité de rationnaliser l'ensemble des équipements, des services et des usages à travers un schéma directeur du numérique pour développer les ressources numériques pédagogiques. II souligne que les aspects techniques des TIC sont utilisés au détriment des aspects pédagogiques et didactiques.

On comprend alors que la disponibilité des technologies à l'école ne garantit pas forcement leur utilisation pédagogique. Ce qui amène Aboubacar \& Adamou (2018) à dire « que si le contexte scolaire nigérien est caractérisé par le souci d'intégrer les TIC dans l'enseignement, les difficultés restent nombreuses pour une utilisation générale et efficiente (...) » (p. 297). Les obstacles pour l'intégration pédagogique des TIC sont, notamment, d'ordre technique et humain. Parmi les obstacles d'ordre technique figurent le manque de connexion internet, le problème d'électricité, le problème de maintenance des appareils et celui de la formation à l'usage pédagogique des TIC. Les obstacles d'ordre humain sont les dispositions psychologiques propres aux utilisateurs/trices tels que : le sentiment d'auto-efficacité, les représentations sociales ou la motivation autodéterminée.

La compréhension des facteurs psychologiques est nécessaire sinon indispensable pour une bonne intégration des technologies à l'école. C'est pourquoi Coulibaly, et al. (2012) ont étudié le sentiment d'auto-efficacité des enseignant-es chercheurs/euses de l'université Abdou Moumouni par rapport à l'utilisation des TIC. À l'aide du Computer Efficacy Scale (CSE) ces auteurs ont formé des groupes d'apprentissage pour la formation des enseignant-es à l'usage des TIC en fonction de leurs niveaux d'habiletés à utiliser ces outils. L'impact du sentiment d'auto-efficacité des enseignant-es du secondaire a aussi fait l'objet d'études de la part de Coulibaly (2009; 2020). Leurs résultats montrent qu'un sentiment d'auto-efficacité élevé influence l'évolution des usages et des pratiques d'intégration pédagogique des TIC.

Les représentations sociales peuvent influer aussi sur l'usage des TIC d'après Kouawo (2011) qui explique que les représentations sociales que l'on développe par rapport à un objet jouent un rôle important dans son acceptation et son usage. Elles permettent selon Kouawo (2011) 
de connaître la manière de penser, de s'approprier, d'interpréter la réalité quotidienne d'un groupe d'individu-es vis-à-vis dudit objet. II affirme qu'au Niger, bien que l'utilisation des TIC à l'école soit récente, les enseignant-es et les élèves développent des représentations sociales favorables à l'égard de ces outils.

Une recherche conduite par Tessa (2011) a révélé l'impact positif des TIC sur la motivation des étudiants à l'apprentissage des mathématiques à l'Université de Niamey.

En parcourant les recherches disponibles à ce jour sur l'usage des TIC en milieu scolaire nigérien, l'on se rend compte qu'elles portent sur des anciennes technologies comme la télévision mais aussi sur l'ordinateur et d'autres outils plus récents. Cependant, celles portant sur le cas spécifique des tablettes sont très rares. Celle réalisée par Baron, et al. (2015) chez les élèves du collège relève de cette dernière catégorie. L'objectif de leur étude était de répondre à la faiblesse des ressources pédagogiques en substituant les livres papiers, et les dépenses associées à ceux-ci, par des ressources numériques pédagogiques. Les résultats indiquent qu'une majorité écrasante d'élèves affirment être très satisfait (70\%) et motivés (80\%) par leurs tablettes numériques.

\section{MÉTHODOLOGIE}

Cette section est consacrée à la méthodologie utilisée pour obtenir les résultats de la présente recherche. Elle précise le champ d'étude ainsi que l'échantillon, les outils de collecte et de traitement des données. L'approche retenue est de type quasi-expérimentale (Campbell \& Stanley, 1963).

\subsection{Champ de l'étude}

Notre champ d'étude est constitué de tous les établissements d'enseignement primaire public de la région de Niamey. Cette dernière est composée de dix-huit (18) inspections reparties sur les cinq (5) arrondissements communaux que compte cette région. Chaque inspection est composée de secteurs pédagogiques auxquels sont rattachées un certain nombre d'écoles primaires. Parmi ces dernières, 10 ont été dotées de tablettes numériques par le projet « Éducation numérique ». Mais pour des raisons techniques (manque d'électricité ; manque de formation des enseignants à l'usage pédagogique du numérique ; affectation des enseignants déjà formés au numérique), sept d'entre elles ont cessé d'utiliser le numérique. C'est de ce terrain que l'échantillon a été tiré.

\section{2. Échantillon}

L'échantillon de l'étude est constitué des élèves des classes de CM2 des trois écoles numériques (publiques) de la région de Niamey, soient 153 individu-es représentant le groupe expérimental. À celles/ceux-là s'ajoute un groupe témoin composé des élèves de trois écoles non numériques (publiques), soit 115 individu-es. Le Tableau 1 ci-dessous montre la répartition 
des groupes. Des codes sont attribués à toutes les écoles pour garantir leur anonymat. Les écoles numériques sont : K1 (avec 39 élèves) ; P3 (44 élèves) et A1 (70 élèves). Quant aux écoles non numériques ce sont : R1 (39 élèves) ; B2 (45 élèves) et Z (33 élèves).

Tableau 1. Effectifs des élèves du CM2 des écoles numériques et non numériques

\begin{tabular}{|l|l|l|l|}
\hline \multicolumn{2}{|c|}{$\begin{array}{c}\text { Écoles numériques } \\
\text { Groupe expérimental }\end{array}$} & \multicolumn{2}{c|}{$\begin{array}{c}\text { Écoles non numériques } \\
\text { Groupe témoin }\end{array}$} \\
\hline K1 & 39 & R1 & 37 \\
\hline A1 & 70 & B2 & 45 \\
\hline P3 & 44 & Z & 33 \\
\hline Total & 153 & Total & 115 \\
\hline
\end{tabular}

Un total de 150 élèves des écoles numériques a été soumis au questionnaire sur l'utilisation pédagogique des tablettes numériques, inspiré de Lestrohan, et al. (2016) (Annexe 1). II a été relevé trois absences (dont une par école) par rapport au premier effectif d'élèves (153) de ce même échantillon. II ressort de ce tableau que la classe A1 est composée d'un effectif de 70 élèves. Ce qui fait d'elle la plus peuplée des deux groupes (groupe expérimental et groupe témoin). Ceci s'explique par le fait que la classe A1 reçoit des élèves venus-es des écoles dites passerelles. Ces dernières relèvent de l'éducation non formelle. Elles accueillent des enfants non scolarisés-es ou déscolarisés-es précocement pour une durée d'étude de 2 ans, à l'issu desquels ces enfants sont reversé-es dans l'éducation formelle en classe de CM2.

\subsection{Scénarios pédagogiques}

Le programme d'études dans toutes les écoles nigériennes a été élaboré en 1988 (réaménagement du programme colonial de 1947). II est dispensé à tous les niveaux de l'enseignement de manière homogène. En dépit des innovations faites à travers l'introduction des TIC (tablettes numériques), le programme reste le même à l'exception des 500 écoles expérimentales bilingues qui développent l'approche par les situations (APS).

Le dispositif d'une classe numérique est composé des tablettes numériques, d'un vidéoprojecteur, d'un écran de projection, d'un serveur et de leurs accessoires. En fonction de l'activité, les tablettes connectées au serveur sont distribuées aux élèves individuellement ou par petits groupes. Pour chaque leçon le contenu à étudier est projeté sur l'écran blanc prévu à cet effet. Les consignes de travail sont dictées par l'enseignante. L'ensemble de la classe participe à la mise en œuvre de l'activité qui est projetée sur l'écran.

Le dispositif d'une classe non numérique est composé des livres pour les élèves, d'un manuel pour l'enseignante, du tableau noir sur lequel le contenu à étudier est porté par la maitresse. En fonction de l'activité, les livres sont distribués par élève ou par groupe. L'enseignante dicte 
les consignes aux élèves. La mise en œuvre de l'activité est assurée sur le tableau par l'ensemble de la classe.

a) Les profils des enseignantes du groupe expérimental

Le tableau 2 ci-dessous présente les profils socioprofessionnels des enseignantes du groupe expérimental. Ces profils sont constitués des variables comme l'âge, le sexe, l'expérience professionnelle et le diplôme ayant permis d'accéder au métier d'enseignant-e.

Tableau 2. Profils des enseignantes du groupe expérimental

\begin{tabular}{|l|l|l|l|l|}
\hline \multicolumn{1}{|c|}{ Enseignantes } & \multicolumn{1}{|c|}{ Âge } & \multicolumn{1}{c|}{ Sexe } & \multicolumn{1}{c|}{ Expérience professionnelle } & Diplôme \\
\hline K1 & 32 & F & 10 ans & CFEEN \\
\hline P3 & 30 & F & 9 ans & CFEEN \\
\hline A1 & 43 & F & 13 ans & CFEEN \\
\hline
\end{tabular}

Le tableau 2 montre que l'âge des enseignantes du groupe expérimental varie entre 32 et 43 ans. La plus expérimentée des enseignantes du groupe dispose de 13 années d'expérience professionnelle. La moins expérimentée dispose de 9 années d'expérience professionnelle. Toutes sont détentrices du Certificat de fin d'études de l'école normale (CFEEN).

b) Les profils des enseignantes du groupe témoin

Le tableau 3 ci-dessous indique les profils des enseignantes du groupe témoin. Comme dans le précédent tableau les variables prises en compte par rapport à ces profils sont l'âge, le sexe, l'expérience professionnelle et le diplôme.

Tableau 3. Profils des enseignantes du groupe témoin

\begin{tabular}{|l|c|c|c|c|}
\hline \multicolumn{1}{|c|}{ Enseignants } & Âge & Sexe & Expérience professionnelle & Diplôme \\
\hline B2 & 39 & F & 16 ans & CFEEN \\
\hline R1 & 43 & F & 15 ans & CFEEN \\
\hline Z & 39 & F & 19 ans & CFEEN \\
\hline
\end{tabular}

À ce niveau, nous constatons que l'âge des enseignantes du groupe témoin varie de 39 à 43 ans. L'enseignante la plus expérimentée dispose de 19 années d'expérience professionnelle. La moins expérimentée dispose de 15 années d'expérience professionnelle. On remarque que dans le groupe expérimental comme dans le groupe témoin, il n'y a aucun enseignant. 


\subsection{Techniques de collecte et d'analyse}

La collecte des données a été réalisée à partir des registres de notes trimestrielles de l'année en cours et d'un questionnaire inspiré de Lestrohan, et al. (2016) administré, à l'aide des tablettes portant le logiciel Kobo Collect, aux élèves des écoles numériques exclusivement. Une comparaison des moyennes issues des résultats des registres de notes, des deux échantillons indépendants - groupe expérimental et groupe témoin- a été effectuée dans un premier temps. Ensuite, dans un second temps, les élèves des écoles numériques (K1, P3 et A1) ont été soumis à un questionnaire portant sur les usages pédagogiques du numérique.

Par ailleurs, nous avons mesuré le sentiment auto-efficacité des enseignantes des deux groupes - expérimental et témoin - à l'aide de l'échelle de Dussault, et al. (2001) adaptée par Coulibaly (2009). Des points (de 1 à 4) sont attribués aux items selon qu'il soit positif ou négatif en fonction des modalités de réponse. Pour les items positifs, la modalité " fortement d'accord » vaut 4 points, alors qu'elle vaut 1 point pour les items négatifs.

Les données de cette collecte ont été analysées à l'aide du calcul de la moyenne et de la variance. Enfin, nous avons établi le profil socioprofessionnel de chaque enseignante.

\section{RÉSULTATS}

Trois types de résultats ont été obtenus dans cette recherche : les résultats issus de l'exploitation des registres des notes des élèves des deux catégories d'écoles (écoles numériques et écoles non numériques), ceux du questionnaire administré aux élèves sur les usages pédagogiques des tablettes et, enfin, ceux de l'échelle du sentiment d'auto-efficacité des enseignantes.

\subsection{Résultats issus de l'exploitation des registres des notes}

Les résultats suivants sont obtenus à la suite de la comparaison des moyennes semestrielles des élèves des écoles numériques (EN) à celles des écoles non numériques (ENN) comme présentés dans le tableau 4 ci-dessous.

Tableau 4. Comparaison de moyennes : écoles numériques et non numériques

\begin{tabular}{|l|c|c|c|}
\hline \multicolumn{1}{|c|}{ Nature de l'établissement } & Moyenne & $\begin{array}{c}\text { Nombre } \\
\text { d'élèves }\end{array}$ & Écart type \\
\hline EN & 7,64 & 150 & 1,53 \\
\hline ENN & 6,79 & 115 & 1,92 \\
\hline Total & 7,27 & 265 & 1,76 \\
\hline
\end{tabular}

I| ressort de ce tableau que les moyennes des élèves des écoles numériques et celles des écoles non numériques sont proches, avec moins d'un point de moyenne de différence. Les 
écarts types de 1,53 et de 1,92 indiquent que la dispersion des notes autour de la moyenne est relativement faible dans les deux groupes. Les résultats scolaires des élèves des écoles numériques $(7,64)$ sont cependant meilleurs que ceux des élèves des écoles non numériques $(6,79)$.

Tableau 5. Moyennes des élèves selon l'établissement d'origine

\begin{tabular}{|l|c|c|c|}
\hline \multicolumn{1}{|c|}{ Établissement } & Moyenne & $\begin{array}{c}\text { Nombre } \\
\text { d'élèves }\end{array}$ & Écart type \\
\hline A1 & 6,84 & 69 & 1,48 \\
\hline B2 & 6,62 & 45 & 2,07 \\
\hline K1 & 8,36 & 38 & 0,80 \\
\hline P3 & 8,28 & 43 & 1,51 \\
\hline R1 & 7,59 & 37 & 1,66 \\
\hline Z & 6,14 & 33 & 1,71 \\
\hline Total & 7,27 & 265 & 1,76 \\
\hline
\end{tabular}

Le tableau 5 présente les moyennes des élèves par établissement, indépendamment des groupes d'appartenance (écoles numériques/écoles non numériques). Nous constatons que les notes varient de 6,14 à 8,28 points sur 10. L'écart type qui est de 1,76, montre que les notes sont concentrées autour de la moyenne $(7,27)$. Sur les 6 écoles testées, les écoles K1 et P3 performent plus que les autres avec respectivement des scores moyens de 8,36 points sur 10 et 8, 28 points sur 10. Ces notes sont au-dessus de la moyenne générale qui est de 7,27 points sur 10. Les écoles $Z$ et B2 sont les moins performantes avec des scores moyens supérieurs au seuil de 5 points sur 10. Ces résultats montrent que, de façon générale, les élèves des écoles numériques réussissent mieux que ceux des écoles non numériques.

Pour voir de plus près le lien entre les résultats scolaires des élèves (moyennes trimestrielles) et la nature de l'établissement (écoles numériques/écoles non numériques), nous avons effectué une analyse de la variance (ANOVA).

Tableau 6. Moyennes des élèves selon l'établissement d'origine

\begin{tabular}{|l|c|c|c|c|}
\hline Source de variation & Ddl & SCE & F & Sig. \\
\hline Entre groupes & 46,349 & 1 & 15,765 &, 000 \\
\hline Intra-groupes & 773,237 & 263 & & \\
\hline Total & 819,586 & 264 & & \\
\hline
\end{tabular}

L'analyse de la variance effectuée dans le tableau 6 ci-dessus montre un coefficient de significativité de 0,000 nettement inférieur au seuil de 5\%. Cela dénote l'existence d'un lien significatif entre la nature de l'établissement et les moyennes des élèves. C'est-à-dire entre les deux groupes comparés (intergroupes). Le fait que les élèves soient issus d'une école 
numérique ou d'une école non numérique influence donc leur réussite scolaire (moyennes trimestrielles).

\subsection{Résultats du questionnaire sur les usages des tablettes numériques}

Le groupe de 150 élèves provenant des classes de CM2 des trois écoles numériques a été soumis au questionnaire pour recueillir les éventuels avantages des tablettes numériques ainsi que leur niveau de satisfaction par rapport aux usages de cet outil. Les usages pédagogiques des tablettes numériques sont analysés à travers des variables comme : la satisfaction à l'égard des tablettes et leurs avantages pédagogiques tels que « rendre le travail en groupe plus facile »; " aider à la progression des apprentissages »; ou " améliorer les relations entre les élèves et l'école.

Tableau 7. Moyennes des élèves selon l'établissement d'origine (en \%)

\begin{tabular}{|l|c|c|c|c|}
\hline \multirow{2}{*}{ Modalités } & \multicolumn{3}{|c|}{ Établissement } & \multirow{2}{*}{ Total } \\
\cline { 2 - 4 } & $\mathrm{A} 1$ & $\mathrm{~K} 1$ & $\mathrm{P} 3$ & 88.70 \\
\hline $\begin{array}{l}\text { Oui, très } \\
\text { satisfait }\end{array}$ & 82.30 & 97.30 & 9.70 & 9.20 \\
\hline $\begin{array}{l}\text { Oui, assez } \\
\text { satisfait }\end{array}$ & 12.90 & 2.70 & 0.00 & 0.70 \\
\hline $\begin{array}{l}\text { Non, pas du } \\
\text { tout satisfait }\end{array}$ & 1.60 & 0.00 & 0.00 & 1.40 \\
\hline Ne sait pas & 3.20 & $0.00 \%$ & 43.00 & 142.00 \\
\hline Total & 62.00 & 37.00 & 100.00 & 100.00 \\
\cline { 2 - 5 }
\end{tabular}

Nous constatons que près de 9/10 (88,7\%) élèves des trois écoles numériques (K1, P3 et $A 1)$ sont «tout à fait satisfait » de l'utilisation des tablettes. Ceci constitue une preuve de l'engouement des élèves pour le numérique. Toutefois, un pourcentage négligeable d'élèves (1\%) affirme ne pas être satisfait de la tablette (Tableau 7). 
Tableau 8. Réponses des élèves sur la facilitation du travail en groupe/établissement (en\%)

\begin{tabular}{|l|c|c|c|c|}
\hline \multirow{2}{*}{ Modalités } & \multicolumn{3}{|c|}{ Établissement } & \multirow{2}{*}{ Total } \\
\cline { 2 - 4 } & $\mathrm{A} 1$ & $\mathrm{~K} 1$ & $\mathrm{P} \mathrm{3}$ & 70.20 \\
\hline Oui, tout à fait & 75.40 & 51.40 & 79.10 & 7.10 \\
\hline Oui, plutôt & 13.10 & 2.70 & 2.30 & 3.50 \\
\hline Non, plutôt pas & 1.60 & 2.70 & 7.00 & 16.30 \\
\hline $\begin{array}{l}\text { Non, pas du } \\
\text { tout }\end{array}$ & 6.60 & 37.80 & 11.60 & 2.80 \\
\hline Ne sait pas & 3.30 & 5.40 & 00 & 100.00 \\
\hline Total & 100.00 & 100.00 & 100.00 & \\
\hline
\end{tabular}

II ressort du tableau 8 que 70,2\% des élèves soutiennent que les tablettes numériques rendent plus facile le travail de groupe, contre 16,3 \% qui déclarent le contraire. L'utilisation de la tablette numérique facilite donc le travail collaboratif d'après la majorité des enquêtés.

Tableau 9. Réponses des élèves sur la progression des apprentissages/établissement (en \%)

\begin{tabular}{|l|c|c|c|c|}
\hline \multirow{2}{*}{ Modalités } & \multicolumn{3}{|c|}{ Établissement } & \multirow{2}{*}{ Total } \\
\cline { 2 - 4 } & $\mathrm{A} 1$ & $\mathrm{~K} 1$ & $\mathrm{P} 3$ & 82.40 \\
\hline Oui, tout à fait & 75.80 & 81.1 & 93.00 & 15.50 \\
\hline Oui, plutôt & 19.40 & 18.90 & 7.00 & 0.70 \\
\hline Non, plutôt pas & 1.60 & 0.00 & 0.00 & 1.40 \\
\hline Ne sait pas & 3.20 & 0.00 & 0.00 & 100.00 \\
\hline Total & 100.00 & 100.00 & 100.00 & \\
\hline
\end{tabular}

Concernant la progression des apprentissages, 97,9\% des élèves soulignent que la tablette les aide à progresser dans l'acquisition des connaissances. Nous pouvons donc dire que cet outil a un fort potentiel pédagogique et que son utilisation impacte positivement la réussite scolaire des élèves. Les résultats montrent en revanche que moins de 1\% des élèves affirment ne pas progresser malgré l'utilisation de tablettes numériques (Tableau 9).

\section{Tableau 10. Réponses des élèves sur l'amélioration des relations élèves et écoles/établissement (en \%)}

\begin{tabular}{|l|c|c|c|c|}
\hline \multirow{2}{*}{ Modalités } & \multicolumn{3}{|c|}{ Établissement } & \multirow{2}{*}{ Total } \\
\cline { 2 - 4 } & $\mathrm{A} 1$ & $\mathrm{~K} 1$ & $\mathrm{P} \mathrm{3}$ & 66.20 \\
\hline Oui, tout à fait & 59.70 & 54.10 & 86.00 & 24.60 \\
\hline Oui, plutôt & 30.60 & 35.10 & 7.00 & 2.10 \\
\hline Non, plutôt pas & 1.60 & 2.70 & 2.30 & 2.80 \\
\hline $\begin{array}{l}\text { Non, pas du } \\
\text { tout }\end{array}$ & 1.60 & 2.70 & 4.70 & \\
\hline
\end{tabular}




\begin{tabular}{|l|c|c|c|c|}
\hline Ne sait pas & 6.50 & 5.40 & 00 & 4.20 \\
\hline Total & 100.00 & 100.00 & 100.00 & 100.00 \\
\hline
\end{tabular}

Le Tableau 10 ci-dessus indique que 90,8\% des élèves interrogés déclarent que le fait d'utiliser les tablettes à l'école améliore leur relation avec celle-ci. Les élèves aiment bien utiliser les tablettes numériques. Et le fait que ces écoles numériques mettent des tablettes à leur disposition renforce le lien entre ces derniers et l'établissement qu'ils fréquentent. Cela peut influencer de façon positive la réussite scolaire des élèves. Néanmoins, 4,2 \% d'entre eux affirment ne rien savoir à ce propos et 4,9 pensent que l'utilisation des tablettes n'améliore pas leur relation avec l'école.

\subsection{Résultats de la mesure du sentiment d'auto-efficacité des enseignantes}

Les résultats de la mesure du sentiment d'auto-efficacité des enseignantes sont présentés en score moyen en fonction des groupes numériques et non numériques. Ensuite, une analyse de la variance intergroupe a été effectuée pour vérifier le lien entre l'utilisation des tablettes et le sentiment d'auto-efficacité des enseignantes.

Tableau 11. Score moyen du sentiment d'auto-efficacité des enseignantes

\begin{tabular}{|l|c|c|c|}
\hline \multicolumn{1}{|c|}{ Type de classe } & Moyennes & N & Écart type \\
\hline Numériques & 50.3333 & 3 & 4.16333 \\
\hline Non numériques & 43.3333 & 3 & 4.16333 \\
\hline Total & 46.8333 & 6 & 5.34478 \\
\hline
\end{tabular}

On remarque que les enseignantes des classes numériques ont un score moyen de 50 points sur 60 au total. Quant aux enseignantes des classes non numériques, elles ont obtenu un score moyen de 43 points sur 60. L'écart type qui est de 5,34 montre que les notes sont concentrées autour de la moyenne (Tableau 11).

Tableau 12. Analyse de la variance intergroupe du sentiment d'auto-efficacité des enseignantes

\begin{tabular}{|c|c|c|c|c|c|c|c|}
\hline & & & $\begin{array}{c}\text { Somme } \\
\text { des } \\
\text { carrés }\end{array}$ & ddl & $\begin{array}{c}\text { Carré } \\
\text { moyen }\end{array}$ & $\mathrm{F}$ & Sig. \\
\hline \multirow{3}{*}{$\begin{array}{l}\text { Score * } \\
\text { Type de } \\
\text { l'école }\end{array}$} & $\begin{array}{l}\text { Entre } \\
\text { groupes }\end{array}$ & \multirow[t]{3}{*}{ (Combinée) } & 73.500 & 1 & 73.500 & \multirow[t]{3}{*}{4.240} & \multirow[t]{3}{*}{.109} \\
\hline & $\begin{array}{l}\text { Intra- } \\
\text { groupes }\end{array}$ & & 69.333 & 4 & \multirow[t]{2}{*}{17.333} & & \\
\hline & Total & & 142.833 & 5 & & & \\
\hline
\end{tabular}


Le Tableau 12 indique l'analyse de la variance entre les groupes. L'analyse de la variance effectuée dans le tableau ci-dessus montre un coefficient de significativité de 0,109 supérieur au seuil de 5\%. Ce qui indique qu'il n'y pas de lien significatif entre la nature de l'établissement et le sentiment d'auto-efficacité des enseignantes. Le fait que les enseignantes soient issues d'une école numérique ou non numérique n'a donc pas de lien avec leurs sentiments d'autoefficacité.

\section{DISCUSSION}

Dans ce travail nous avons tout d'abord comparé les moyennes semestrielles des élèves de deux catégories d'écoles. Les premières utilisent le numérique en classe tandis que les secondes ne l'utilisent pas. Les résultats de cette comparaison vont en faveur des écoles numériques. Ces résultats confirment ceux de Suppert, et al. (2019) qui ont montré qu'une utilisation fréquente des tablettes dans les tâches éducatives conduit à un meilleur rendement scolaire en mathématiques et en français et cela grâce à une forte motivation autodéterminée et de meilleures perceptions de compétence.

En revanche, ces résultats contredisent ceux de Dauphin (2016) qui trouve que l'utilisation de l'iPad en classe se traduit par une baisse générale des résultats scolaires. Nos résultats sont aussi différents de ceux de Aboubacar \& Adamou (2018) qui n'ont trouvé en contexte nigérien aucune différence entre les résultats scolaires des élèves utilisant le numérique et ceux qui ne l'utilisent pas.

Ensuite les résultats du questionnaire adressé aux élèves révèlent qu'une grande majorité d'entre eux souligne leur satisfaction par rapport à l'usage pédagogique des tablettes numériques. Cet usage leur procure des avantages comme la facilitation du travail en groupe, la facilitation des apprentissages, et l'amélioration de la relation élèves/écoles. Ces résultats vont dans le sens de l'étude de Colegessi \& Balleux (2018) qui fait ressortir les avantages pédagogiques des tablettes numériques. Ils relèvent trois plus-values des pratiques pédagogiques avec les tablettes. II s'agit de la différenciation pédagogique, de la créativité et de la construction de synthèses.

Nos résultats vont aussi dans le même sens que ceux de Karsenti \& Fievez (2013) qui ont relevé 15 avantages de l'utilisation pédagogique des tablettes numériques. Les avantages comme la motivation accrue des élèves ; l'accès à l'information ; l'organisation du travail plus aisée ; la collaboration accrue entre les élèves sont proches des avantages que nous avons identifiés à savoir la satisfaction à l'égard des tablettes, la facilitation du travail en groupe et la progression des apprentissages des élèves. Nos résultats concordent enfin avec ceux de Lestrohan, et al. (2016) sur la satisfaction des utilisateurs/trices quant à une utilisation pédagogique des tablettes numériques. 


\section{CONCLUSION}

Ce travail a un double objectif : d'une part celui de mesurer l'impact de l'utilisation des tablettes numériques sur la performance scolaire des élèves et d'autre part celui d'identifier les avantages de l'usage pédagogique des tablettes en classe.

La comparaison des moyennes trimestrielles des deux groupes d'écoles révèle que les performances scolaires des élèves des écoles numériques sont meilleures que celles des écoles non numériques. L'ANOVA confirme ces résultats en indiquant que l'utilisation des tablettes numériques impacte positivement les performances des élèves.

II ressort de l'analyse descriptive que la quasi-totalité des élèves soulignent leur satisfaction par rapport à l'utilisation des tablettes numérique tout en indiquant les avantages pédagogiques de cet outil. Rechercher, de manière fine et à l'aide d'approches qualitatives quels sont les avantages pédagogiques de la tablette numérique dans ce contexte nigérien permettrait d'affiner les résultats obtenus ici. De plus, au vu de ces résultats il serait judicieux d'engager d'autres recherches quantitatives à l'aide d'évaluations standardisées ou des recherches mixtes et/ou longitudinales sur des échantillons plus larges afin de mieux comprendre la situation dans le contexte du Niger et plus particulièrement de sa capitale, Niamey.

\section{RÉFÉRENCES}

Aboubacar, Y. I., \& Adamou, B. (2018). TIC et performances des élèves maitres des Écoles normales d'instituteurs au Niger. European Scientific Journal, 14(25), 293-307.

Awokou, K. (2007). De l'utilisation des médias et des technologies de l'information et de la communication dans l'éducation de 1960 à 2006. Le cas du Togo. Thèse de doctorat, Université de Rouen. https://tel.archives-ouvertes.fr/tel-00139109

Baron, G-L., Zablot, S., Combemorel-Pauty, C., \& Le Quentrec, E. (2015). Bilan et perspectives de l'expérimentation «Tablettes » au Niger. Paris : Université Paris Descartes - Orange Labs research.

Cabinet du Premier Ministre. (2014). Programme sectoriel de l'éducation et de la formation, PSEF. 2014-2024. Niamey : République du Niger.

Cabinet du Premier Ministre. (2018). Rapport d'Etat sur le système éducatif nigérien, RESEN. Niamey : République du Niger.

Cabinet du Premier Ministre. (2020). Programme transitoire sectoriel de l'éducation et de la formation, PTSEF. 2020-2022. République du Niger.

Campbell, D., \& Stanley, J. (1963). Experimental And Quasi-experimental Designs For Research. Chicago, IL: Rand McNally.

Colognesi, S., \& Balleux, L (2018). Former les futurs instituteurs primaires à utiliser les tablettes numériques dans leur classe : une spécialisation optionnelle en haute " école pédagogique ». Frantice.net, 14, 7-24. 
Coulibaly, M. (2009). Impact des TIC sur le sentiment d'auto-efficacité des enseignants du secondaire au Niger et leur processus d'adoption d'une innovation. Thèse de doctorat, Université de Montréal].

Coulibaly, M. (2012). Sentiment d'auto-efficacité à l'égard des TIC : processus d'adoption de ces technologies dans l'enseignement secondaire au Niger. Saabrucken (Allemagne) : Éditions universitaires européennes.

Coulibaly, M. (2020). Auto-efficacité à l'égard de l'ordinateur chez des enseignants du secondaire au Niger. In T. Karsenti, K. Toure, M. Lepage, \& S. A. Attenoukon, Usages et appropriation des technologies éducatives en Afrique : quelques pistes de réflexion (pp. 79100). Bamenda : Langaa.

Dauphin, A. (2016) Tablettes numériques au secondaire : quel impact sur les résultats scolaires ? https://www.researchgate.net/publication/309286337

Depover, C., Karsenti, T., \& Komis, V. (2007). Enseigner avec les technologies : favoriser les apprentissages, développer des compétences. Québec : Presses de l'Université du Québec.

Dessus, P. (2015). L'effet des médias sur l'apprentissage. Grenoble: Université Grenoble Alpes.

Fiévez, A., \& Karsenti, T. (2018). Usages et perceptions des enseignants lors de l'utilisation de la tablette en contexte scolaire. Formation et profession, 26(1), 55-73. http://dx.doi:10.18162/fp.2018.394

Galy, K. A., \& Dané, É. (2003). Obstacles et solutions à l'appropriation des NTICs au Sud : leçons sur l'expérience de l'Université Abdou Moumouni du Niger. http://www.francophonie-durable.org/documents/colloque-ouaga-a4-galy.pdf

Halilou Garba, M., Issaka Moussa, F., \& Adamou, B. (2020). Influence des langues utilisées dans l'enseignement de base 1 sur les performances des élèves des Collèges d’Enseignement Général de Niamey, Niger. Revue francophone d'éducation comparée (RAFEC), 6, 247-260.

Inaudi, A. (2017). École et numérique : une histoire pour préparer demain. Hermès, 2(78), $72-$ 79. https://www.cairn.info/revue-hermes-la-revue-2017-2-page-72.htm

Karsenti, T., \& Fievez, A. (2013). L'iPad à l'école : usages, avantages et défis. Résultats d'une enquête auprès de 6057 élèves et 302 enseignants du Québec (Canada). Rapport préliminaire des principaux résultats. http://www.karsenti.ca/ipad/pdf/rapport_iPad_Karsenti-Fievez_FR.pdf

Karsenti, T., \& Fievez, A. (2013). Les tablettes tactiles à l'école primaire : avantages, défis et recommandations pour les enseignants. AQEP : Vivre le primaire - Motivation et apprentissage, 26(4), 33-36.

Kouawo, A. (2011). Que pensent les enseignants et les élèves du secondaire des TIC ? Une étude des représentations sociales au Niger. Thèse de doctorat, Université de Montréal. https://papyrus.bib.umontreal.ca/.../Kouawo_Achille_2011_these.pdf

Laferrière, T., Bader, B., Barma, S., Beaumont, C., DeBlois, L., Gervais, F., ... Lessard, A. (2011). L'étude de la réussite scolaire au Québec : une analyse historico-culturelle de 
I'activité d'un centre de recherche, le CRIRES. Éducation et francophonie, 39(1), 156-182. https://doi.org/10.7202/1004335ar

Legendre, R. (1993). Éducation numérique. Dans dictionnaire actuel de l'éducation. Montréal, QC : Guérin.

Lestrohan, E., Boisson, L., \& Tardieu, B. (2016). L'utilisation des équipements individuels mobiles au collège de Sèvres. https://docplayer.fr/69277982-L-utilisation-desequipements-individuels-mobiles-au-college-de-sevres.html

Ministère de l'éducation de base [MEBA]. (2003). Programme décennal de développement de l'éducation : 2003-2013. Niamey : République du Niger.

Ministère de l'enseignement primaire de l'alphabétisation, de la promotion des langues nationales et de l'éducation civique. [MEP/A/PLN/EC]. (2016). Statistique de l'éducation de base et alphabétisation. Niamey : République du Niger.

Ministère de l'enseignement primaire de l'alphabétisation, de la promotion des langues nationales et de l'éducation civique [MEP/A/PLN/EC]. (2018a). Rapport d'État sur le Système Éducatif du Niger (RESEN). Niamey : République du Niger.

Ministère de l'enseignement primaire, de l'alphabétisation, de la promotion des langues nationales et de l'éducation civique [MEP/AVPLN/EC]. (2018b). Rapport d'évaluation des compétences des enseignants contractuels du primaire. Niamey : République du Niger.

Ministre du Plan (2010). Programme de développement économique et social, PDES. Niamey : République du Niger.

Moeglin, P. (2017). Du manuel scolaire aux systèmes numérisés d'information et de communication : le cas de l'école primaire. Hermès, 2(78), 65-71.

Programme d'analyse des systèmes éducatifs de la CONFEMEN [PASEC]. (2016). Performance du système éducatif nigérien : compétence et facteurs de réussite au primaire. Dakar : CONFEMEN/PASEC.

Rabardel, P. (1995). Les hommes et les technologies : Approche cognitive des instruments contemporains. Paris: Armand Colin.

Sagayar, M. (2018). Les ressources numériques : analyse des pratiques, représentations, besoins et attentes des usagers dans les Universités publiques du Niger. Réseau africain francophone d'éducation comparée (RAFEC), 5, 309-329.

Supper, W., Guay, F., Falardeau, E., Karsenti, T., \& Pelletier, G. (2019). La fréquence d'utilisation de la tablette numérique à des fins pédagogiques et le rendement scolaire des élèves : le rôle médiateur de la motivation autodéterminée. Revue canadienne de l'éducation, 42(2), 517-552.

Tessa, O. (2011). Impacts des TIC sur la motivation des étudiants à l'apprentissage des mathématiques à l'Université Abdou Moumouni au Niger. Thèse de doctorat, Université de Montréal.

Tiemtoré, Z. (2018). L'enseignement face au défi de la qualité : quelles attitudes adoptées entre théories nouvelles et pratiques figées ? Réseau africain francophone d'éducation comparée (RAFEC), 5, 297-309.

Villemonteix, F., \& Khaneboubi M. (2013). Étude exploratoire sur l'utilisation d'iPads en milieu scolaire : entre séduction ergonomique et nécessités pédagogiques. Sciences et 
Technologies de l'Information et de la Communication pour l'Éducation et la Formation, 20, 445-464. https://doi.org/10.3406/stice.2013.1078

Villemonteix, F., \& Khaneboubi, M. (2012, Septembre). Utilisations de tablettes tactiles à l'école primaire. Journées Communication et Apprentissage Instrumentés en Réseau, Septembre 2012, Amiens, France. https://edutice.archives-ouvertes.fr/edutice-00765323

Open Access Publications - Bibliothèque de I'Université de Genève Creative Commons Licence 4.0

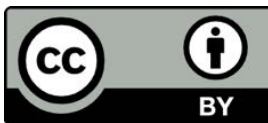

